\title{
A Study for Obtaining Weights in Pairwise Comparison Matrix in AHP
}

\author{
Hyeong Chul Jeong ${ }^{1} \cdot$ Jong Chan Lee ${ }^{2} \cdot$ Myoungshic Jhun ${ }^{3}$ \\ ${ }^{1}$ Department of Applied Statistics, University of Suwon \\ ${ }^{2}$ Department of Statistics, Korea University \\ ${ }^{3}$ Department of Statistics, Korea University
}

(Received February 1, 2012; Revised March 7, 2012; Accepted April 16, 2012)

\begin{abstract}
In this study, we consider various methods to estimate the weights of a pairwise comparison matrix in the Analytic Hierarchy Process widely applied in various decision-making fields. This paper uses a data dependent simulation to evaluate the statistical accuracy, minimum violation and minimum norm of the obtaining weight methods from a reciprocal symmetric matrix. No method dominates others in all criteria. Least squares methods perform best in point of mean squared errors; however, the eigenvectors method has an advantage in the minimum norm.
\end{abstract}

Keywords: AHP, Pairwise comparison matrix, Eigenvector method, Distance least squares, minimum norm.

\section{1. 서론}

Saaty $(1980,2003)$ 에 의해 개발된 계층분석적 의사결정론(Analytic Hierarchy Process; AHP)은 의 사결정의 목표 혹은 평가기준이 복합적인 경우, 상호배반적인 대안들을 체계적으로 평가하여 합리적 의사결정을 수행할 수 있도록, 대안의 순서를 결정하는 의사결정 지원 체계의 한 방법이다. 일반적으 로 AHP 분석은 복잡한 문제를 비교적 단순한 계층구조화하여 주요요인과 세부요인으로 나눈 후, 이 들 요인들의 쌍대비교(pairwise comparison)를 통하여 중요도(혹은 가중치)를 도출하는 과정을 따른 다. 즉, $\mathrm{AHP}$ 방법은 계층구조설정 $\rightarrow$ 가중치 추정 및 일관성 검증 $\rightarrow$ 평점(measurement) $\rightarrow$ 환 류(feedback)의 과정을 거치는 의사결정론이다 (Basak, 1989; Donegan과 Dodd, 1992).

본 연구는 AHP 의사결정론의 4 단계 중 한 과정인 가중치 추정 문제를 다루었다. AHP 과정에서 순 위결정행렬이라 불리는 쌍대비교행렬 $A$ 에 근거하여 가중치 벡터 $w$ 를 계산하는 방법은 크게 세 가지 로 분류된다. 즉, (1) 고유치분해법 (2) 거리최소화법 (3) 평균계산법 등이다 (Jeong, 2011; Gass와 Rapcsak, 2004; Zahedi, 1986). 이들 방법 중 Saaty (1980)의 고유벡터법, Cogger와 Yu (1985)의 수정 고유벡터법, Gass와 Rapcsak (2004)의 SVD 방법 등 고유치분해법이 다른 추정방법에 비해 AHP과정

This research was supported by Basic Science Research Program through the National Research Foundation of Korea(NRF) funded by the Ministry of Education, Science and Technology(2010-0008825).

${ }^{1}$ Corresponding author: Associate Professor, Department of Applied Statistics, University of Suwon, Hwaseong, Gyeonggi, 445-743, Korea. E-mail: jhc@suwon.ac.kr 
에서 주로 선호된다. 이는 Saaty (1980)가 처음 계층적 의사결정론을 제안하면서, 특별한 이론적 근거 는 없지만 가중치(모수) 추정방법으로 고유치분해법을 사용하였기 때문에, 다수의 학자들이 의사결정론 으로 $\mathrm{AHP}$ 를 수용하면서 가중치 계산방법 역시 같이 수용하였기 때문으로 생각된다. 하지만, 최근에 와 서 고유벡터 방법이 Perron-Frobenius 정리라는 양행렬(positive matrix) 이론에 수학적 근거가 있음이 보여짐으로, 고유치분해법의 수리적 근거가 좀 더 보강되고 있는 실정이다 (Kinoshita, 2008).

한편, 거리최소화법 역시 직접최소제곱법, 가중최소제곱법, 로그최소제곱법, 로그절대오차최소법 등 다 양한 방법이 존재한다. 이들은 고유치분해법과는 달리 자료와 가중치와의 오차를 최소화하는 통계적 관 점에서 개발된 추정방법들이다. 또한, 평균계산법은 이들 고유치분해법이나 거리최소화법에 비해 쌍대 비교행렬의 특징을 사용하여 비교적 간략한 해를 유도하는 과정에서 나온 계산방법들이라 할 수 있다.

그런데, 지금까지의 많은 연구결과에 의하면, 어느 하나의 가중치 추정 방법이 다른 방법에 비해 우위 에 있는 방법은 없다고 알려진다 (Golany와 Kress, 1993; Gass와 Rapcsak, 2004; Kumar와 Ganesh, 1996). 즉, 최소제곱법에 의한 중요도 가중치의 근거를 능가할 고유치 모델은 없으며, 고유치 모델의 중요도 가중치를 능가할 최소제곱법은 없다는 것이 정설이다. 하지만, 위에 언급한 바처럼 고유치분해 법이 많은 사람들의 지지를 얻는 편이지만, 이에대한 반박도 많은 편이다 (Costa와 Vansnick, 2008; Golden과 Wasil, 2003). 고유치분해법은 수리적 간결성으로 인하여 현실 문제 해결로 선호되지만, 통 계적(stochastic) 관점보다는 확증적(deterministic) 관점에 서있는 방법이다. 그러므로 통계적 관점에 서 보면 최소제곱법이 고유치분해법에 비해 더 좋은 장점을 다수 지니고 있다. 이는 모수와 추정치와의 거리를 측정 평가 기준으로 선택하는 평균제곱오차(MSE)라는 통계적관점에서, 최소제곱법이 고유치모 델보다 우수함을 의미한다 (Zahedi, 1986).

본 연구는 기존의 여러 추정방법을 가능한 다수 소개하고, MSE 이외 다양한 모형 평가방법을 소개하기 로 한다. 특히, 최소노름 평가기준을 제시하고, 다양한 평가기준에 의한 가중치 추정결과를 비교하기로 한다. 추정량의 비교를 위해 분포의존적인 모의실험을 실시할 수 있는데, 쌍대비교행렬에 오차행렬을 삽입시키는 모의실험은 오차행렬과 비일치성을 유발시키는 정도와의 관계가 우선 규명되어야 하는 문제 가 있으므로, 본 연구에서는 참값이 알려진 상황에 대한 자료의존적 모의실험을 실시하였다.

2 장에서는 쌍대비교행렬의 일반적 성질을 소개하였으며 3 장에서는 11 가지 추정방법을 소개하였다. 4 장 에서는 평가방법을 소개하였다. 또한 5 장에서는 자료의존적 모의실험으로 이들 방법을 비교하였다.

\section{2. 쌍대비교 행렬의 이론적 근거}

$\mathrm{AHP}$ 에서 얻어지는 쌍대비교행렬 $A=\left(a_{i j}\right)$ 에 대해 (1) $a_{i j}>0,(2) a_{i j}=1 / a_{j i}, 1 \leq i \leq j \leq n$, (3) $a_{i j}=1$ 의 성질이 성립한다. 특히, (2)의 성질을 만족하는 행렬을 역수행렬(reciprocal matrix)이라고 한다. 행렬의 각 원소가 양의 값인 행렬을 양행렬(positive matrix), 비음인 행렬을 비 음행렬(nonnegative matrix)이라 하면, $\mathrm{AHP}$ 의 쌍대비교행렬 $A$ 는 대각성분이 1 인 역수대칭양행 렬(reciprocal symmetric positive matrix)이다. 그리고, $A$ 에 기초하여 가중치 혹은 순위를 결정하 므로 $\mathrm{AHP}$ 의 쌍대비교행렬 $A$ 를 가중(순위)결정행렬이라고도 한다.

정의 2.1 행렬 $A=\left(a_{i j}\right)$ 의 각 원소가 $a_{i j} a_{j k}=a_{i k} ; i, j, k=1, \ldots, n$ 를 만족하면 일치성(CONSISTENT)이 있다 하고, 행렬 $A$ 를 일치행렬(CONSISTENCY MATRIX)이라 한다.

쌍대비교에 의해서 얻어진 행렬 $A$ 의 원소 $a_{i j}$ 가 각각 $w_{i} / w_{j}$ 의 값을 가지고 있다면 기수적 일관성, 즉, $a_{i j} \times a_{j k}=a_{i k}$ 가 성립되어야 한다. $a_{i j} \times a_{j k}=a_{i k}$ 의 의미는 ' $i$ 를 $j$ 보다 $x$ 배 중요하게 생각하고 $j$ 는 $k$ 보다 $y$ 배 중요하게 생각한다면, $i$ 는 $k$ 보다 $x \times y$ 배 중요하게 평가한다'는 뜻을 지니고 있다. 그런데, 
$\mathrm{AHP}$ 가중치 추정 문제는 위의 일치성이 지켜지지 않기에 발생한다. 순위결정행렬에 대해 다음과 같은 Perron의 정리가 있다 (Kinoshita, 2008).

정리 2.1 (PERRON의 정리) $A$ 가 양행렬이고 $A$ 의 고유치 집합을 $L$ 이라 하면,

(1) $\lambda_{\max }=\max \{|\lambda| \mid \lambda \in L\}$ 이 되는 양의 고유치 $\lambda_{\max } \in L$ 이 존재한다.

(2) $\lambda_{\max }$ 에 대응하는 양의 고유벡터 $u$ 가 존재하고, 양의 고유벡터는 $u$ 의 상수배이다.

$$
n_{t+1}=\left(\begin{array}{c}
n_{0, t+1} \\
n_{1, t+1} \\
n_{2, t+1} \\
\vdots \\
n_{k, t+1}
\end{array}\right)=\left(\begin{array}{ccccc}
f_{0} & f_{1} & f_{2} & \cdots & f_{k} \\
p_{0} & 0 & 0 & \cdots & 0 \\
0 & p_{1} & 0 & \cdots & 0 \\
& & & \vdots & \\
0 & 0 & 0 & p_{k-1} & 0
\end{array}\right)\left(\begin{array}{c}
n_{0, t} \\
n_{1, t} \\
n_{2, t} \\
\vdots \\
n_{k, t}
\end{array}\right)=M n_{t}
$$

Bernadelli (1941)과 Leslie (1945)는 동물의 개체연구에서 $t+1$ 시점에서 $i$ 연령에 해당하는 동물의 개 체수 $n_{i, t+1}$ 의 관계를 연구하였는데, $t$ 시점에 생존한 동물의 개체 수 $n_{i, t}$ 가 출생한 개체를 고려하여 생 존하는 빈도 $f_{i}$ 및 $t$ 시점에서 $t+1$ 시점까지 생존할 확률 $p_{i}$ 에 영향을 받는 모형으로 식 (2.1)의 행렬식 을 제안한 바 있다. 여기서, 앞의 연구자들은 $M n_{T}=\lambda n_{T}$ 를 만족하는 벡터 $n_{T}$ 가 존재하는 가에 관심 을 두었다. 이는, 만약 $\lambda=1$ 이라면, $t+1$ 시점에도 $t$ 시점과 동일한 수의 개체가 유지되는 $t$ 시점의 개 체수를 찾는 문제와 동일하게 된다.

식 (2.1)과 같은 문제는 $\mathrm{AHP}$ 의 가중결정행렬에도 그대로 적용된다. $\mathrm{AHP}$ 에서 '평가행렬의 주 고유벡 터가 중요도 의미를 지닌다'라는 고유벡터법은 '각 항목은 스스로의 가치를 평가하고 그 평가치를 결정 한다'라는 전제조건이 포함되어있다. 즉, $i$ 항목이 스스로의 가치를 평가하여 그 평가치를 $w_{i}$ 로 놓으 면, 이 $w_{i}$ 를 $i$ 항목의 자기평가치라 부를 수 있다는 점이다. 또한, $n$ 개의 항목으로 구성된 행렬 $A$ 의 $(i, j)$ 요소 $a_{i j}$ 는 $j$ 항목의 가치에 대한 $i$ 항목 가치의 비교를 나타내고 있다. 이것에 의해 $a_{i j} w_{j}$ 는 $j$ 항목 $w_{j}$ 로 자기자신을 평가할 때의 $i$ 항목 가치 $w_{i}$ 와 대등하다. 즉, $i$ 항목의 $j$ 항목으로부터 $a_{i j} w_{j}$ 라 고 추정된 것이다. 이에따라 $i$ 항목은 자기 이외의 $n-1$ 개의 항목에서 그 가치가 평가되는 과정을 따르고 있다. 그러므로 $w_{i}$ 는 $i$ 항목의 자기평가치가 되며, $\sum_{j \neq i} a_{i j} w_{i}$ 는 $i$ 항목의 외부평가치가 된다 (Kinoshita, 2008).

Bernadelli (1941) 등의 동물개체 유지 수준의 의미를 여기에 적용하면, 자기평가치와 외부평가치의 평 균이 일정한 상태를 유지하는 수준 값이 가중결정행렬에서 가중치 벡터를 찾는 문제와 동일함을 짐작할 수 있다. 만일, 자기평가치와 외부평가치의 평균이 일치하는 것을 희망한다면,

$$
\frac{1}{n-1} \sum_{j \neq j} a_{i j} w_{j}=w_{i}
$$

의 관계를 도출하는 문제로 전환된다. 즉, $w_{i} \sum_{j \neq j} a_{i j} w_{j} /(n-1)$ 은 $i$ 항목에 대한 외부평가와 내부평 가의 비로 과잉평가율의 의미를 지니게 되며, 이는 오차의 개념을 띄게 된다. 즉, 과잉평가율의 오차를 억제하는 문제를 다음과 같이 표현할 수 있다.

$$
\min \max \left[\frac{\sum_{j \neq 1} a_{1 j} w_{j}}{(n-1) w_{1}}, \ldots, \frac{\sum_{j \neq n} a_{n j} w_{j}}{(n-1) w_{n}}\right] \quad \text { s.t } w_{1}>0, \ldots, w_{n}>0 .
$$

이에 대해 다음의 Frobenius Min-Max 정리가 존재한다. 
정리 2.2 (Frobenius Min-Max 정리) $B=\left(b_{i j}\right)$ 를 비음행렬이라 할 때, $B$ 의 최대고유치 $\lambda_{\max }$ 에 대해 다음이 성립한다. 또한, 모든 $w_{i}>0$ 이 성립한다.

$$
\max \min \left[\frac{\sum_{j=1}^{n} b_{1 j} w_{j}}{w_{1}}, \ldots, \frac{\sum_{j=1}^{n} b_{n j} w_{j}}{w_{n}}\right]<\lambda_{\max }<\min \max \left[\frac{\sum_{j=1}^{n} b_{1 j} w_{j}}{w_{1}}, \ldots, \frac{\sum_{j=1}^{n} b_{n j} w_{j}}{w_{n}}\right]
$$

또한, 행렬 $B$ 가 기약행렬이면, 주고유벡터 $\bar{W}=(\bar{w})$ 에 대해 다음의 식이 성립한다.

$$
\max \min \left[\frac{\sum_{j=1}^{n} b_{1 j} \bar{w}_{j}}{\bar{w}_{1}}, \ldots, \frac{\sum_{j=1}^{n} b_{n j} \bar{w}_{j}}{\bar{w}_{n}}\right]<\lambda_{\max }<\min \max \left[\frac{\sum_{j=1}^{n} b_{1 j} \bar{w}_{j}}{\bar{w}_{1}}, \ldots, \frac{\sum_{j=1}^{n} b_{n j} \bar{w}_{j}}{\bar{w}_{n}}\right] .
$$

$\mathrm{AHP}$ 가중결정행렬을 $1 /(n-1)[A-I]$ 로 나타낼 수 있는데, 이는 기약행렬이며, 비음행렬이므로 Frobenius 정리를 적용하면,

$$
\frac{1}{n-1}[A-I] w=1 \cdot w
$$

이 된다.

이는 고유치 1 에 대한 고유벡터를 찾는 문제로 귀결된다. 즉, 여기서 유도된 주 고유벡터가 AHP 행렬 의 가중치로 결정되는 것이 고유치분해법의 이론적 근거가 된다고 할 수 있다. 즉, 고유치분해에 의한 가중치 결정방법은 구해야할 가중치에 사전정보를 제약조건으로 포함시킴으로 최적해가 존재하게 되는 체계이다. 만일 제약조건이 포함되지 않는다면, 최적해는 일치하지 않게 된다. 이러한 점이 고유치분해 법의 장점인과 동시에 약점이 될 수도 있다 (Kinoshita, 2008).

Saaty (2003)는 본인이 주장한 고유치 이론의 또 다른 배경으로 순위보전(order preservation)을 제시 하였다. 순위보전은 다음과 같은 성질을 의미한다.

$w_{i} \geq w_{j}$ 이면, $(A)_{i} \geq(A)_{j}$ 이며, $(A e)_{i} \geq(A e)_{j}$ 이고,

$$
\lim _{p \rightarrow \infty} \frac{1}{p} \sum_{m=1}^{p} \frac{\left(A^{m} e\right)_{i}}{e^{\prime} A^{m} e} \geq \lim _{p \rightarrow \infty} \frac{1}{p} \sum_{m=1}^{p} \frac{\left(A^{m} e\right)_{j}}{e^{\prime} A^{m} e}
$$

이다. 여기서, $(A)_{i}$ 는 행렬 $A$ 의 $i$ 번째 행을, $(A e)_{i}$ 는 행렬 $A$ 의 $i$ 번째 행의 합이며, $e=[1,1, \ldots, 1]^{\prime}$ 이 다. 만일 $A w=\lambda_{\max } w$ 가 성립하면, 행렬 $A$ 의 순위보전이 성립됨으로 고유치분해법의 이론적 정당성이 부여된다. 또한, Saaty (2003)의 순위보전 증명과정에

$$
\lim _{k \rightarrow \infty} \frac{A^{k} e}{\lambda_{\max }^{k}}=c w, \quad \text { 여기서 } c \text { 는 임의의 상수 }
$$

를 Sylvester's formula를 사용하여 전개하였는데, 이와같은 성질은 지배고유치(dominant eigenvalues)가 있다면,

$$
\frac{b_{i, k}}{b_{i, k-1}}=\frac{\theta \lambda_{\max }^{k} u_{i 1}}{\theta \lambda_{\max }^{k-1} u_{i 1}}=\lambda_{\max }
$$

을 유도하는 문제와 동일하다. 여기서, $A=U D U^{-1}$ 와 0 이 아닌 벡터 $x$ 에 대해 $b_{k}=\lambda_{1}^{k} u_{1} v_{1}^{\prime} x, \theta=$ $v_{1}^{\prime} x$ 를 의미한다. 즉, 지배고유치이론은 고유치와 고유벡터를 행렬계산을 하지 않고 유도할 수 있는 멱 등법(power method)의 이론적 근거가 된다. 


\section{3. 추정방법}

\section{1. 고유치분해방법}

1) 고유벡터 방법(Eigenvector Method; EM)

고유벡터 방법은 Saaty의 전통적 방법으로 쌍대비교 행렬 $A$ 를 고유치 분해에 의해 $\lambda_{\max }$ 에 대응되는 고 유벡터 $w$ 의 표준화벡터를 가중치로 사용하는 방법이다.

$$
A \hat{w}=\lambda_{\max } \hat{w}, \quad 1^{\prime} \hat{w}=1 .
$$

여기서, $A$ 가 비일치행렬이면, $\lambda_{\max }>n$ 이 성립하며, $n$ 은 행렬 $A$ 의 차원이다.

2) 수정 고유벡터 방법(Modified Eigenvector Method; MEM)

Cogger와 $\mathrm{Yu}$ (1985)에 의해 제안된 수정 고유벡터 방법은 $T=\left[t_{i j}\right]$ 를 $A$ 의 상삼각행렬, $G=\left[g_{i i}\right]$, $g_{i i}=n-i+1$ 를 대각행렬이라 놓고, $A$ 가 일치행렬일 때,

$$
T w=G w, \quad G^{-1} T w=1^{\prime} \cdot w
$$

의 성질을 이용하는 방법이다. 실제 계산에서는 $G^{-1} T$ 를 고유치분해하여 가중치를 계산한다. 여기서 가중치는

$$
\hat{w}_{i}=\sum_{j=i+1}^{n} \frac{a_{i j} \cdot \hat{w}_{j}}{n-1}, \quad i=1, \ldots, n-1,1^{\prime} \hat{w}=1
$$

로 계산되어 각 행의 위치에 따라 추정치가 영향을 받는 단점이 존재한다 (Takeda 등, 1987).

3) 멱승법(Power Method; PM)

멱승법은 지배고유치 이론에 근거하여 고유치 분해를 하지 않고도 근사 고유벡터와 근사 고유치를 얻는 방법이다. 멱승법에 의한 가중치 벡터 $w$ 를 계산하는 절차는 다음과 같다.

(1) 초기 벡터를 $w(0)=[1 / n, 1 / n, \ldots, 1 / n]$ 으로 놓는다.

(2) $k=1,2, \ldots$ 에 대해, $w(k)=A^{k} w(k-1), 1^{\prime} w(k)=1$ 를 계산한다.

(3) $|w(k)-w(k-1)| \leq \epsilon$ 이면, $\hat{w}=w(k)$ 이다.

4) 특이값 분해(Singular Value Decomposition Method; SVD)

비교행렬 $A$ 의 특이값 분해를 통해 $w$ 를 추정한다. 모수벡터 $w$ 의 추정값 $\hat{w}$ 은 행렬 $A$ 의 좌고유벡터 $u=\left(u_{1}, \ldots, u_{n}\right)$ 와 우고유벡터 $v=\left(v_{1}, \ldots, v_{n}\right)$ 에 대해

$$
\hat{w}_{i}=\frac{u_{i}+1 / v_{i}}{\sum_{j=1}^{n}\left(u_{j}+1 / v_{j}\right)}, \quad i=1, \ldots, n
$$

로 계산한다 (Gass와 Rapcsak, 2004). 


\section{2. 거리최소화법}

1) 로그최소제곱법(Logarithmic Least Squares Method; LLS)

$a_{i j}=\left(w_{i} / w_{j}\right) \epsilon_{i j}$ 에 대해, 양변에 $e$ 를 밑으로 하는 로그를 취하면,

$$
\log a_{i j}=\log w_{i}-\log w_{j}+\log e_{i j}
$$

이다. 여기서, $E\left[\log e_{i j}\right]=0, \operatorname{Var}\left[\log e_{i j}\right]=\sigma^{2}$ 이다. 이제 Gauss Markov 정리를 적용하면 최소제곱추 정치 $\hat{w}_{i}$ 가 $\log w_{i}$ 의 최량추정량(best estimator)이 된다. 비일치행렬 $A$ 에 대해 로그최소제곱법을 적용 하여 최적해를 구하려면 $\sum \log w_{i}=0$ 의 제약조건이 필요하다. 즉,

$$
D\left(a_{i j}, \frac{w_{i}}{w_{j}}\right)=\left\|\log a_{i j}-\log \left(\frac{w_{i}}{w_{j}}\right) 0\right\|
$$

에 대해, 로그최소제곱법은

$$
\min \sum_{i} \sum_{j}\left(\log a_{i j}-\log w_{i}+\log w_{j}\right)^{2} \quad \text { s.t } \prod_{i=1}^{n} w_{i}=1
$$

이며 가중치 벡터는 다음과 같다.

$$
\hat{w}_{i}=\prod_{j=1}^{n}\left(a_{i j}\right)^{\frac{1}{n}}
$$

2) 직접최소제곱법(Direct Least Squares Method; DLS)

직접최소제곱법은 로그변환하지 않고 유클리디안 거리를 최소화하는 아래의 방정식의 해를 가중치로 사 용하는 방법이다.

$$
\min \sum_{i=1}^{n} \sum_{j=1}^{n}\left(a_{i j}-\frac{w_{i}}{w_{j}}\right)^{2} \quad \text { s.t } \quad \sum_{i=1}^{n} w_{i}=1 .
$$

위의 방정식은 수치해석으로 풀어야 하는데, 종종 유일해가 존재하지 않는 경우가 발생한다.

3) 가중최소제곱법(Weighted Least Squares Method; WLS)

가중최소제곱법은 최소제곱법과 유사하나, 직접최소제곱법이 다수의 해를 취할 수 있는 반면, 가중최소 제곱법은 유일해를 취한다. 가중최소제곱법은 다음의 식을 최소화 하는 해를 계산한다.

$$
\min \sum_{i=1}^{n} \sum_{j=1}^{n}\left(a_{i j} w_{j}-w_{i}\right)^{2} \quad \text { s.t } \quad \sum_{i=1}^{n} w_{i}=1 .
$$

실제 계산에서는 라그랑쥐법을 사용한 선형방정식

$$
\sum_{i \neq k}^{n}\left(a_{i k}^{2}+n-1\right) w_{k}-\sum_{j \neq k}^{n}\left(a_{j k}+a_{k j}\right) w_{j}+\lambda=0, \quad \sum_{i=1}^{n} w_{i}=1
$$

의 유일해 $\hat{w}_{i}$ 를 계산하면 된다. 


\section{3. 평균법}

1) 평균치환법(Mean Transformation Method; MTM)

평균치환법은 가중결정행렬 $A=\left(a_{i j}\right)$ 의 전치행렬 $A^{\prime}$ 의 행 원소를 행의 합으로 나눈 행렬 $B=\left(b_{i j}\right)$ 를 계산한 후 다음과 같이 가중치를 추정한다.

$$
\hat{w}_{j}=\sum_{i=1}^{n} \frac{b_{i j}}{n}, \quad j=1, \ldots, n .
$$

2) 기하평균법(Geometric Mean Method; GM)

행 기하평균법은 $\hat{w}_{i}=\prod_{j=1}^{n}\left(a_{i j}\right)^{1 / n}$ 이며, 열 기하평균법은 $\hat{w}_{j}=1 / \prod_{i=1}^{n}\left(a_{i j}\right)^{1 / n}$ 으로 가중치를 추정 한다. 여기서 행 기하평균법은 로그최소제곱법과 동일하다. 그런데, Zahedi (1986)는 AHP의 쌍대비 교행렬이 역수대칭하지 않는 행렬을 full 행렬이라 하였다. Full 행렬에서는 행 기하평균과 열 기하평균 값이 서로 다르나, 역수대칭행렬에서는 행렬의 성질에 의해 두 값이 서로 동일하다.

3) 조화평균법(Harmonic Mean Method; HMM)

조화평균법은 좌고유벡터 방법으로 $\hat{w} A=\lambda_{\max } \hat{w}$ 와 같이 가중치를 추정한다 (Johnson 등, 1979). 실제 계산은 $A^{\prime} \hat{b}=\lambda_{\max } \hat{b}$ 의 최대고유벡터에 대해 $\hat{w}=1 / \hat{b}, 1^{\prime} \hat{w}=1$ 으로 해를 구한다. 여기서 계산된 $\hat{w}$ 들은 각 대안들의 조화평균 형태를 따른다.

4) 단순평균법(Simple Row Average Method; SRA)

단순평균법은 $\hat{w}_{i}=\left(\sum_{j=1}^{n} a_{i j}\right) / n$ 으로 가중치를 계산한다.

\section{4. 평가}

가중결정행렬에 기초하여 가중치를 추정하는 다양한 방법에 대한 성능을 평가하는 연구는 매우 흥미로 운 문제이다. 추정치의 성능을 평가하는 방법으로 적절한 오차를 일치행렬에 삽입하여 비일치행렬을 생 성한 후 비일치행렬에서 추정한 가중치와 일치행렬의 모수와의 차이(평균제곱오차)를 비교하는 것이 일 반적으로 생각할 수 있는 평가방법이다. 그런데, 의사결정관점에서는 일치행렬과 비일치행렬과의 차이 를 비교하는 것보다 비일치행렬 내의 불균형 정도를 최소화시키는 방법이 더 좋은 추정방법일 수 있다. 이는 $\mathrm{AHP}$ 행렬의 각 원소 $a_{i j}$ 가 오차 $e_{i j}$ 가 추가된 $a_{i j}=\left(w_{i} / w_{j}\right) e_{i j}$ 로 관찰된다는 사실에 기초하여, $a_{i j} w_{j}$ 는 $i$ 항목에 대한 자기평가치이고 $n-1$ 회의 $i$ 항목에 대한 평가 $\sum_{j \neq i} a_{i j} w_{j}$ 는 $i$ 항목에 대한 외부 평가치의 합이라는 점을 고려할 때, 내부평가와 외부평가가 균형을 이루는 상태로 가중치를 추정하는 방 법이 보다 좋을 수 있음을 의미한다. 이러한 관점에 기초하여 $\mathrm{AHP}$ 의 가중치 추정 평가에 대해 다음의 $L_{1}, L_{2}$ 노름을 주요한 평가 기준으로 제안하고자 한다.

$$
\begin{array}{ll}
L_{1}=\sum_{i=1}^{n}\left|\frac{\sum_{j \neq i} a_{i j} w_{i j}}{n-1}-w_{i}\right|, & \bar{L}_{1}=\sum_{t=1}^{T} \frac{L_{1 t}}{T} \\
L_{2}=\sum_{i=1}^{n}\left(\frac{\sum_{j \neq i} a_{i j} w_{i j}}{n-1}-w_{i}\right)^{2}, & \bar{L}_{2}=\sum_{t=1}^{T} \frac{L_{2 t}}{T}
\end{array}
$$


여기서, $L_{1}, L_{2}$ 노름은 의사결정단계에서 자기평가와 외부평가의 비차의 오차를 최소화하는 추정방법에 유리한 도구로 정리 2.2에서 언급한 Frobenius Min-Max 정리와 관련되어 있다. 해당 정리에 의해, 제 안한 평가 방법들은 고유치분해방법들에 다소 유리할 수 있음을 짐작할 수 있다.

다음으로 추정방법의 성능을 평가하는 도구로 다음의 분산과 평균제곱오차을 고려하였다.

$$
\begin{aligned}
\operatorname{Var}\left(\hat{w}_{i}\right)=\sum_{t=1}^{T} \frac{\left(\hat{w}_{i t}-\bar{w}_{i}\right)^{2}}{T-1}, & i=1, \ldots, n ; & \operatorname{Var}(\hat{\mathbf{w}})=\sum_{i=1}^{n} \operatorname{Var}\left(\hat{w}_{i}\right), \\
\operatorname{MSE}\left(\hat{w}_{i}\right)=\sum_{t=1}^{T} \frac{\left(\hat{w}_{i t}-w_{i}\right)^{2}}{T}, & i=1, \ldots, n ; & \operatorname{MSE}(\hat{\mathbf{w}})=\sum_{i=1}^{n} \operatorname{MSE}\left(\hat{w}_{i}\right) .
\end{aligned}
$$

위와 같은 기준들은 행렬 $A=\left[a_{i j}\right]$ 와 참값 $w_{i}$ 와의 오차제곱을 최소화하는 거리최소화법에 유리한 평가 도구임을 짐작할 수 있다.

한편, Golany와 Kress (1993)는 위반(violation) 개념을 고려하였다. 대안 $i$ 가 대안 $j$ 보다 선호되면 $w_{i}>w_{j}$ 가 유지되어야 하지만, 최소위반(minimum violation)이란 이러한 순위가 유지되지 않는 경우 를 의미한다. 최소위반은 다음과 같이 계산한다.

$$
\mathrm{MV}_{t}=\sum_{i} \sum_{j} \frac{I_{i j}(t)}{2}, \quad t=1, \ldots, T
$$

여기서, $I_{i j}$ 는 $a_{i j}<1$ 일 때 $\hat{w}_{i}>\hat{w}_{j}$ 이거나, $a_{i j}>1$ 일 때 $\hat{w}_{i}<\hat{w}_{j}$ 이면, $I_{i j}(t)=1 ; a_{i j} \neq 1$ 일 때 $\hat{w}_{i}=\hat{w}_{j}$ 이거나, $a_{i j}=1$ 일 때 $\hat{w}_{i} \neq \hat{w}_{j}$ 이면, $I_{i j}(t)=1 / 2$; 그 이외의 경우 $I_{i j}(t)=0$ 인 지시함수이다.

결론적으로 본 연구에서는 평가도구로, 내외부 균형상태, 평균제곱오차, 최소위반이라는 크게 3 가지 평 가도구를 고려하였다. 통계적으로는 참값과 차이를 최소화하는 추정방법이 중요한 평가기준이 되지만, 제안한 $L_{1}, L_{2}$ 노름과 같이 제로합(zero-sum) 2 인 게임에서 안장점(saddle point)을 찾는 것과도 관련 있는, 균형상태를 유지하는 평가 기준이 필요하다는 것을 강조하고자 한다.

\section{5. 모의실험}

\section{1. 자료 및 가중치 추정}

다음 실험은 기초통계학 강의를 듣는 $\mathrm{H}$ 대학교 학생 70 명에게 도형의 크기의 비를 추정하게 한 실험으 로, Saaty (2003)의 실험을 재현한 것이다. 학생들은 Figure 5.1을 보고 10 문항의 쌍대비교 질문지에 응답하였다. 설문 문항은 일반적인 AHP 방법론에 의해 1-9점 척도로 측정되었다 (Jeong, 2010). 측정 된 자료를 역수대칭행렬로 변화한 후 앞의 11 가지 방법으로 각각 가중치를 추정하였다. 제시된 각 도형 의 실제 크기의 비는 $w=[0.47,0.05,0.24,0.14,0.09]$ 이다.

$$
A=\left(\begin{array}{ccccc}
1 & 9 & 2 & 3 & 4 \\
1 / 9 & 1 & 1 / 4 & 1 / 2 & 1 \\
1 / 2 & 4 & 1 & 2 & 3 \\
1 / 3 & 2 & 1 / 2 & 1 & 1 \\
1 / 4 & 1 & 1 / 3 & 1 & 1
\end{array}\right)
$$

Figure 5.1은 한 명의 실험자로부터 얻은 식 (5.1)의 쌍대비교행렬 $A$ 에 대한 각 방법의 추정 결과로 3 장 의 순서로 제시되어 있다. 여기서 CGM은 열 기하평균법을 의미한다. 


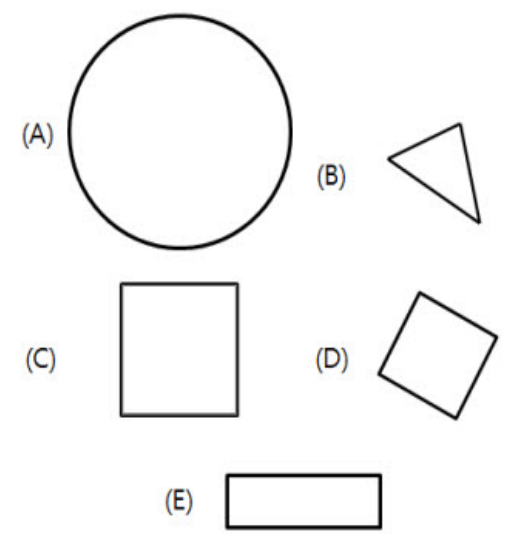

Figure 5.1. Five figures presented for simulation study

Table 5.1. Evaluation results using reciprocal symmetric positive matrix of (5.1)

\begin{tabular}{c|ccccccccc}
\hline Method & $w_{1}$ & $w_{2}$ & $w_{3}$ & $w_{4}$ & $w_{5}$ & MSE & MV & $L_{1}$ & $L_{2}$ \\
\hline EM & 0.4584 & 0.0669 & 0.2538 & 0.1250 & 0.0959 & 0.0009 & 1 & 0.0191 & 0.0001 \\
MEM & 0.4687 & 0.0723 & 0.2551 & 0.1020 & 0.102 & 0.0023 & 0.5 & 0.0468 & 0.0010 \\
PM & 0.4584 & 0.0669 & 0.2538 & 0.1250 & 0.0959 & 0.0009 & 1 & 0.0192 & 0.0001 \\
SVD & 0.4633 & 0.0552 & 0.2371 & 0.1409 & 0.1035 & 0.0003 & 1 & 0.0763 & 0.0014 \\
LLS & 0.4571 & 0.0663 & 0.2564 & 0.1252 & 0.0949 & 0.0009 & 1 & 0.0165 & 0.0001 \\
DLS & 0.4671 & 0.0563 & 0.2414 & 0.1327 & 0.1024 & 0.0003 & 1 & 0.0638 & 0.0009 \\
WLS & 0.4571 & 0.0538 & 0.2489 & 0.1377 & 0.1024 & 0.0004 & 1 & 0.0520 & 0.0007 \\
MTM & 0.4550 & 0.0675 & 0.2549 & 0.1251 & 0.0975 & 0.0010 & 1 & 0.0250 & 0.0003 \\
CGM & 0.4571 & 0.0663 & 0.2564 & 0.1252 & 0.0949 & 0.0009 & 1 & 0.0165 & 0.0001 \\
HMM & 0.4559 & 0.066 & 0.2592 & 0.1253 & 0.0937 & 0.0011 & 1 & 0.0209 & 0.0001 \\
SRA & 0.4659 & 0.0702 & 0.2575 & 0.1185 & 0.0879 & 0.0012 & 1 & 0.0302 & 0.0002 \\
\hline
\end{tabular}

식 (5.1) 행렬에 대한 일치도 값 $\mathrm{CI}=\left(\lambda_{\max }-n\right) /(n-1)=0.019$ 로 비교적 높은 일치성을 보이고 있다. Table 5.1에서 보면, 고유벡터방법 $(\mathrm{EM})$ 과 멱승법 $(\mathrm{PM})$ 의 가중치 추정결과치가 일치하는데, 이는 멱승 법이 Saaty의 고유벡터방법의 수렴치에 해당하기 때문이다. 본 예에서는 4 회의 반복에 의해 수렴하였 다. 또한, 로그최소제곱법 $(\mathrm{LLS})$ 과 열기하평균법 $(\mathrm{CGM})$ 의 해가 같음을 볼 수 있는데, 이는 식 (5.1)의 자료행렬이 full 행렬이 아닌 역수대칭행렬이기 때문이다. Table 5.1의 추정값들이 참 값에 상당히 가까 움을 볼 수 있다. 흥미로운 점은 이들 11 개의 추정치의 평균이 $[0.46,0.06,0.25,0.13,0.10]$ 으로 모수와 매우 일치한다는 점이다.

\section{2. 모의실험 결과}

Table 5.2 는 70 명의 자료에 근거한 평가 결과이다. 여기서 70 명의 평균 $\mathrm{CI}$ 는 0.045 로 집단의 일관성 은 높은 편이다. CI의 분포와 집단의 의사결정에 대해서는 Lee (2012)의 결과를 참고할 수 있다. 멱승 법 $(\mathrm{PM})$ 은 평균 4.43 회 수렴으로 고유벡터방법 $(\mathrm{EM})$ 과 일치된 결과를 유도하였으며, 언급한 바와 같이 역수대칭행렬이므로 LLS와 CGM은 동일한 결과를 유도하였다.

Table 5.2의 결과를 살펴보자. MSE가 가장 작은 방법은 직접최소제곱법(DLS)이며, EM은 낮은 성능 
Table 5.2. Simulation results based on 70 data (The numbers in ( ) refer to priority orders for various criteria.)

\begin{tabular}{c|ccccc}
\hline Method & Variance & MSE & MV & $\bar{L}_{1}$ & $\bar{L}_{2}$ \\
\hline EM & $0.01088(8)$ & $0.01564(10)$ & $0.79286(5)$ & $0.04524(1)$ & $0.00175(1)$ \\
MEM & $0.01152(9)$ & $0.01521(7)$ & $0.47143(1)$ & $0.08008(9)$ & $0.00650(10)$ \\
PM & $0.01087(7)$ & $0.01563(9)$ & $0.79286(5)$ & $0.04525(2)$ & $0.00175(1)$ \\
SVD & $0.01258(11)$ & $0.01638(11)$ & $1.10714(11)$ & $0.08917(11)$ & $0.00562(9)$ \\
LLS & $0.00941(4)$ & $0.01386(4)$ & $0.76429(2)$ & $0.04585(3)$ & $0.00294(4)$ \\
DLS & $0.00924(3)$ & $0.01330(1)$ & $1.05000(10)$ & $0.08718(10)$ & $0.00505(8)$ \\
WLS & $0.01157(10)$ & $0.01522(8)$ & $0.99286(9)$ & $0.06772(7)$ & $0.00286(3)$ \\
MTM & $0.00911(2)$ & $0.01422(6)$ & $0.79286(5)$ & $0.05544(6)$ & $0.00467(7)$ \\
CGM & $0.00941(4)$ & $0.01386(4)$ & $0.76429(2)$ & $0.04585(3)$ & $0.00294(4)$ \\
HMM & $0.00950(6)$ & $0.01371(3)$ & $0.76429(2)$ & $0.05140(5)$ & $0.00333(6)$ \\
SRA & $0.00850(1)$ & $0.01360(2)$ & $0.80000(8)$ & $0.07997(8)$ & $0.00737(11)$
\end{tabular}

을 보이고 있다. 또한, 수정고유벡터방법(MEM)이나 특이값분해(SVD)의 MSE가 낮음을 볼 수 있다. 이와 같은 결과는 앞선 많은 연구에서, $\mathrm{MSE}$ 관점에서는 최소제곱법이 고유치분해법보다 우수한 결과를 유도한다는 것과 일치된 결과이다 (Kumar와 Ganesh, 1996; Saaty와 Vargas, 1984). 거리최소제곱법 중 특히 직접최소제곱법(DLS)이 가장 오차가 작으며, 다음으로 로그최소제곱법(LLS)의 오차가 작음을 볼 수 있다. 특이한 점은 위의 자료에 대해 평균치환법 $(\mathrm{MTM})$, 단순평균법(SRA) 등 평균법도 $\mathrm{MSE}$ 관점에서 비교적 좋은 성능을 보이고 있다는 점이다.

최소위반 관점에서는 $\mathrm{MEM}$ 이 다른 방법에 비해 가장 우수한 성능을 보이고 있으며 SVD나 DLS는 최 소위반 관점에서는 가장 좋지 못하다. 사실, 가중치라는 것이 0 과 1 사이의 작은 값에서 결정되기에 각 대안들의 가중치 값의 차이가 매우 미비할 수 있다. 그러므로 의사결정과정에서는 실제 가중치 값보다 각 대안들의 순위에 더 관심이 있을 수 있다. 즉, $n$ 개 대안에 대한 가중치보다 각 대안의 등수를 기초로 의사결정의 순서를 정하는 경우가 많기 때문이다. 이에 따라 최소위반 $(\mathrm{MV})$ 평가치가 경우에 따라 중요 한 지표가 될 수 있다. 흥미로운 점은 $\mathrm{MSE}$ 관점에서는 $\mathrm{DLS}$ 가 좋으나, 최소위반 관점에서 $\mathrm{DLS}$ 가 좋지 못한 상반된 결과가 유도되었다는 점이다.

이제 본 연구에서 관심을 둔 $L_{2}$ 노름을 살펴보자. $L_{2}$ 노름은 자기평가와 외부평가가 균형을 이루는 추 정치를 찾는 방법이다. 여기서 보면, Saaty의 고유벡터방법(EM)이 다른 방법에 비해 가장 $L_{2}$ 노름이 작음을 발견할 수 있다. 이에 반해 SRA나 DLS 방법은 가장 노름이 커져서 자기평가와 외부평가의 비 대칭이 커지고 있음을 볼 수 있다.

결론적으로 평가를 어떤 방법으로 하느냐에 따라 항상 우수한 통계량은 존재하지 않는다. 참 값에 가까 운 가중치를 얻고자 한다면, 직접최소제곱법(DLS) 등 최소제곱법이 우수하며, 최소위반 관점에서는 수 정고유벡터방법 $(\mathrm{MEM})$ 이 우수하다. 하지만, 게임이론의 균형상태를 유지하는 평가치를 얻고자 한다면 Saaty의 고유벡터방법(EM)이 우수함을 모의실험에서 볼 수 있다. 하지만, 로그최소제곱법(LLS)은 어 느 경우나 가장 좋은 값은 주지는 못하지만, 각 평가에서 항상 상위권에 있는 방법임을 볼 수 있다.

\section{6. 결론}

본 연구는 의사결정론에서 일반적으로 널리 사용되는 AHP 방법 중 가중결정행렬에서 가중치를 추정하 는 방법을 소개하고 여러 평가기준을 제안 및 소개하였다. 이를 기초로 참값이 알려진 상황에서 모의실 험을 통하여 여러 추정방법을 비교하였다. 연구의 한계는 쌍대비교행렬에 오차를 개입하여 비일치성행 
렬을 생성하지 않고 설문조사에 의한 실험에 의해 얻어진 비일치행렬을 사용한 점이다. 또한, full 행렬 이 아닌 역수대칭행렬을 사용하였으며, 비교적 낮은 차원의 실험으로 제한되어 고차원에서 추정방법의 특징을 살펴보지 못한 점에 한계를 둘 수 있다. 하지만, 본 연구를 통해 여러 추정방법을 소개하였고, 새 로운 평가도구를 언급하였으며, 모의실험을 통해 통계적 관점에서는 최소제곱법이 우수하지만, 균형상 태의 의사결정을 유도하기 위해서는 그 방법론의 단순성으로 많은 비판의 대상이었던 Saaty의 고유벡터 법이 역시 우수하다는 점을 살펴본 것에 연구의 의의를 둘 수 있다.

$\mathrm{AHP}$ 에서는 본 연구에서 언급한 추정방법 이외 추정된 값에 대한 구간추정, 검정론, 오차행렬의 분포 및 집단의사결정에 의한 추정방법 및 검정론 등의 많은 문제가 존재한다. 추후 $\mathrm{AHP}$ 의 통계적 이론에 대한 고찰이 필요하다.

\section{References}

Basak, I. (1989). Estimation of the multi-criteria worths of the alternatives in a hierarchical structure of comparisons, Communication in Statistics Theory and Methods, 18, 3719-3738.

Bernadelli, H. (1941). Population waves, Journal of the Burma Research Society, 31, 1-18.

Cogger, K. O. and Yu, P. L. (1985). Eigen weight vectors and least distance approximation for revealed preference in pairwise weight ratios, Journal of Optimization Theory and Applications, 36, $483-491$.

Costa, C. B. and Vansnick, J. (2008). A critical analysis of the eigenvalue method used to derive priorities in AHP, European Journal of Operational Research, 187, 1422-1428.

Donegan, H. A. and Dodd, F. J. (1992). A new approach to AHP decision-making, The Statistician, 41, $295-302$.

Gass, S. I. and Rapcsak, T. (2004). Singular value decomposition in AHP, European Journal of Operational Research, 154, 573-584.

Golany, B. and Kress, M. (1993). A multicriteria evaluation of methods for obtaining weights from ratio-scale matrices, European Journal of Operational Research, 69, 210-220.

Golden, B. and Wasil, E. A. (2003). Celebrating 25 years of AHP based decision making, Computers and Operational Research, 30, 1419-1497.

Jeong, H. C. (2010). Study on AHP and non-parametric verification on the importance of the diagnosis indicators of personal information security level, Journal of the Korean Data Analysis Society, 12,3(B), 1499-1510.

Jeong, H.C. (2011). A note for obtaining weights from pairwise comparison matrix, Preceeding of the Journal of the Korean Data Analysis Society.

Johnson, C. R., Beine, W. B. and Wang, T. J. (1979). A note on right-left asymmetry in an eigenvector ranking procedure, Journal of Mathematical Psychology, 19, 61-64.

Kinoshita, E. (Kang, J. K. and Min, B. C.) (2008). AHP Theory and Practice, Intervision.

Kumar, N. and Ganesh, L. S. (1996). A simulation-based evaluation of the approximate and the exact eigenvector methods employed in AHP, European Journal of Operational Research, 95, 656-662.

Lee, J. C. (2012). A Study on the Statistical Property of AHP, A Doctoral Dissertation, Korea University.

Leslie, P. H. (1945). On the use of matrices in certain population mathematics, Biometrika, 33, 183-212.

Saaty, T. L. (1980). The Analytic Hierarchy Process, McGraw-Hill, New York.

Saaty, T. L. (2003). Multicriteria Decision Making: The Analytic Hierarchy Process, McGraw-Hill, New York.

Saaty, T. L. and Vargas, L. G. (1984). Comparison of eigenvalue, logarithmic least squares and least squares methods in estimating ratios, Mathematical Modelling, 5, 309-324.

Takeda, E., Cogger, K. and Yu, P. L. (1987). Estimating criterion weights using eigenvectors: A comparative study, European Journal of Operational Research, 29, 360-369.

Zahedi, F. (1986). A simulation study of estimation methods in the analytic hierarchy process, SocioEconomic Planning Sciences, 20, 347-354. 